\title{
A Case of Acute Telogen Effluvium After SARS-CoV-2 Infection
}

\author{
Shuying $\operatorname{Lv} \mathbb{D}^{1,2}$ \\ Lei Wang ${ }^{2}$ \\ Xiaohui Zou ${ }^{3}$ \\ Zihan Wang (D) \\ Baoquan $\mathrm{Qu}^{1,2}$ \\ Wenjun Lin ${ }^{1,2}$ \\ Dingquan Yang ${ }^{2}$
}

'School of Clinical Medicine, Beijing University of Chinese Medicine, Beijing, People's Republic of China; ${ }^{2}$ Department of Dermatology, China-Japan Friendship Hospital, Beijing, People's Republic of China; ${ }^{3}$ Laboratory of Clinical Microbiology and Infection, Department of Pulmonary and Critical Care Medicine, China-Japan Friendship Hospital, Beijing, People's Republic of China
Correspondence: Dingquan Yang Department of Dermatology, China-Japan Friendship Hospital, Beijing, People's Republic of China

Tel +86 I390I2l867|

Emailydqlx@163.com
Abstract: As the number of COVID-19 cases increasing, more and more patients are concerning about alopecia, a sequela after SARS-CoV-2 infection. We here report a case of a 38-year-old woman with a typical acute telogen effluvium (ATE) after recovery from COVID-19.

Keywords: COVID-19, SARS-CoV-2, acute telogen effluvium, alopecia

\section{Case Presentation}

On January 29, 2020, a woman aged 38 years old presented fever and cough but she did not visit a doctor. When she visited Beijing Haidian Hospital on February 8, she was diagnosed with COVID-19 and admitted to the hospital. On February 21, she recovered and was discharged from the hospital. Up to April 30, she visited our department with complaint about severe hair loss in the past week ( $>150$ hairs/day), as well as oily scalp and trichodynia. Dermatology examination revealed diffuse hair loss involving the entire scalp. There was no obvious patchy hair loss area, and the forehead hairline was not significantly receded (Figure 1A). The pull test was positive in the whole head. Dermoscopy showed scalp inflammation, capillarectasia, dandruff, increased density of telogen hairs, and relatively uniform diameter of the terminal hair shaft. There was no broken hair, black dots, exclamation point hairs, etc (Figure 2A). The pigmentation at the proximal end of hair root was reduced, and the end of hair root was clubbed (Figure 3). Blood tests excluded vitamin B12, trace elements deficiencies, autoimmunity, and thyroid dysfunction. Therefore, the patient was diagnosed as acute telogen effluvium. Topical 5\% minoxidil, mixed with halcinonide solution, was sprayed evenly on the hair loss area 1-2 times a day for treatment. Meanwhile, selenium sulfide lotion mixed with shampoo 2-3 $\mathrm{mL}$ was used to wash hair 2-3 times a week. After 3 months of treatment, the patient felt hair loss was significantly alleviated; symptoms of oily scalp and trichodynia disappeared. The hair pull test turned negative, and many new hairs grew out (Figures 1B and 2B). The study protocol was approved by the Medical Ethical Committee of China-Japan Friendship Hospital, and written informed consents were obtained from the patient to publish the case details.

\section{Discussion}

Acute telogen effluvium (ATE) is clinically manifested as diffuse alopecia of the whole head with a sudden increase in hair loss. The clinical manifestation of this "the pain in the hair" are named trichodynia. The onset and progression of hair loss 


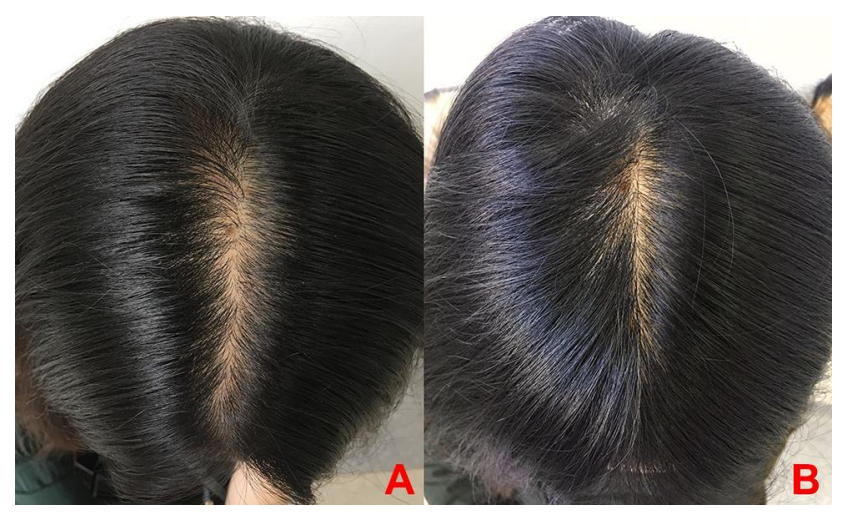

Figure I Head hair at initial diagnosis $(\mathbf{A})$ and 3 months later (B).

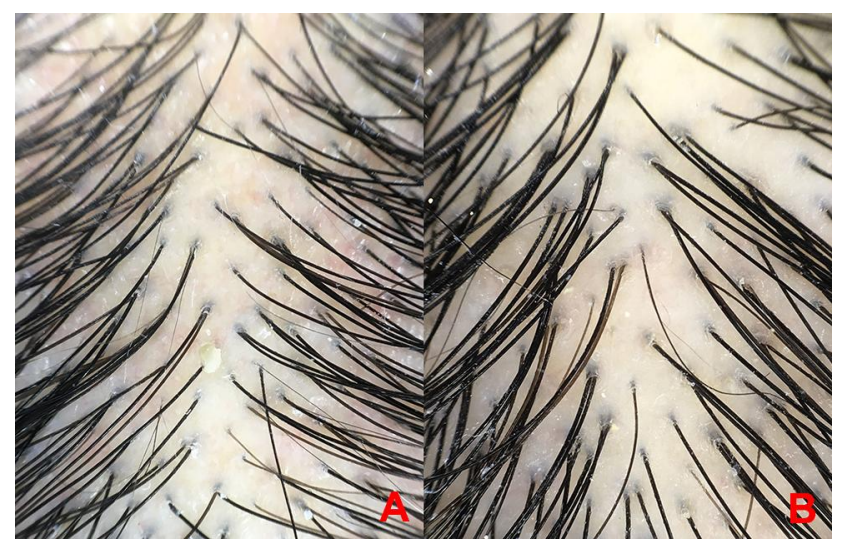

Figure 2 Trichoscopic graph at initial diagnosis (A) and 3 months later (B).

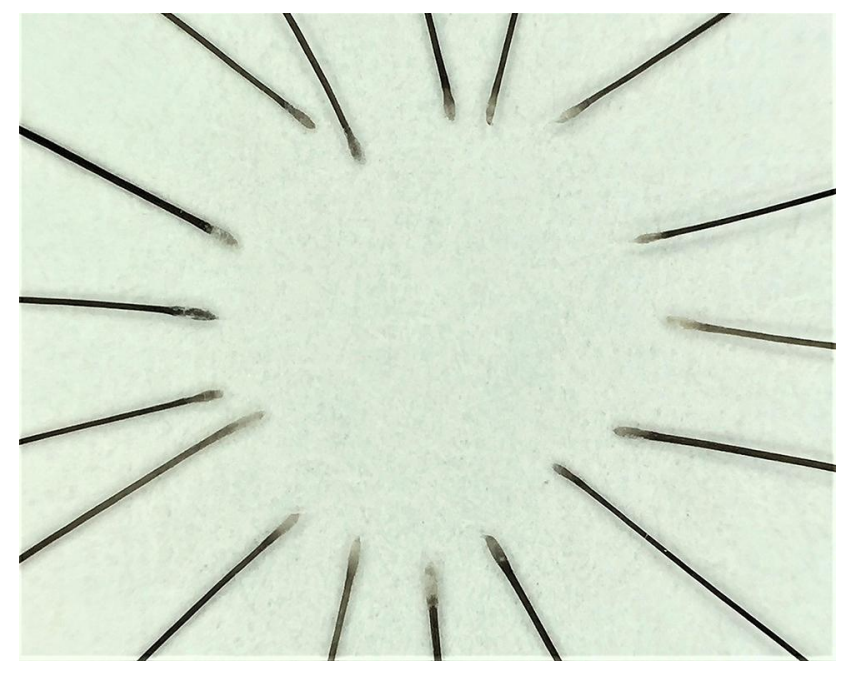

Figure 3 Trichoscopy presentation of hair roots.

is rapid, usually with positive result of hair pull test. ATE usually lasts less than 6 months.

The typical trichoscopic features of ATE include some hair follicles without hair shaft, increased density of telogen hairs, and relatively uniform diameter of the terminal hair shaft. The proximal end of loosen hairs' roots are clubbed, and the pigmentation of them are reduced. ${ }^{1}$ In this case, the patient's trichoscopy showed scalp inflammation, capillarectasia, dandruff, many telogen hairs and relatively uniform diameter of the terminal hair shaft. The patient's hair loss was diffuse and did not restricted to the top of the head. Thus, this case is distinguished from diffuse alopecia areata and female-pattern androgenetic alopecia.

First reported by Kligman ${ }^{2}$ in 1961, ATE is a noncicatricial alopecia disease characterized by the simultaneous loss of many telogen hairs. Based on the duration of the disease, it can be divided into acute telogen effluvium (ATE) and chronic telogen effluvium (CTE). ATE usually occurs 2 to 3 months after a trigger event, which includes postpartum, discontinuation of birth control pills, hyperthermia, malnutrition, bulimia, blood loss, shock, surgery, and severe psychological factors, etc. Previous studies had observed telogen effluvium (TE) occurred after dengue virus and rickettsia conorii infection. ${ }^{3,4}$ Recent studies also showed that some patients developed hair loss after SARS$\mathrm{CoV}-2$ infection. In an investigation of clinical sequelae of COVID-19 survivors in Wuhan, China, ${ }^{5}$ the prevalence of alopecia as a sequela was as high as $48.5 \%$. However, the exact mechanism between COVID-19 and TE is unclear. A prospective study ${ }^{6}$ enrolled 214 patients with ATE from March 2020 to August 2020, of whom 89.7\% (191 patients) had a confirmed diagnosis of prior SARS-CoV-2 infection. The case we reported here suggests that SARS-CoV-2 infection may also be one of the causes of ATE.

Hoffmann et $\mathrm{al}^{7}$ demonstrated that SARS-CoV-2 used the SARS-CoV receptor ACE2 for entry and the serine protease TMPRSS2 for S protein priming. Therefore, any cell that carries the ACE2 receptor is a potential target for SARS-CoV-2, including the skin. A recent $\mathrm{study}^{8}$ has detected SARS-CoV-2 in the sweat glands and sweat ducts of COVID-19 patients. To further clarify the specific mechanism of TE following COVID-19 infection, additional testing of hair follicle tissue is required.

What makes this TE case different from the previous ones is that in the era of COVID-19 pandemic, TE has become a common sequela, which seriously affects the psychosocial condition of patients recovered from COVID-19. Also, the COVID-19 epidemic has an impact on people's social life, and some are more prone to anxiety and depression, from which TE may occur. Turkmen et $\mathrm{al}^{9}$ investigated the effect of COVID-19 pandemic on hair disorders through online questionnaire, which showed that $27.9 \%$ of participants experienced TE during the pandemic. A retrospective study 
in Turkey ${ }^{10}$ showed that the percentage of TE in dermatology clinics increased 5.51 times during the pandemic by comparing with the same season of the previous year. Therefore, we need to link TE with this emerging and highly impactful disease and further investigate the specific mechanisms by which COVID-19 may cause TE.

\section{Consent}

The patient in this manuscript has given written informed consent to the publication of her case details.

\section{Funding}

There is no funding to report.

\section{Disclosure}

The authors report no conflicts of interest in this work.

\section{References}

1. Zhu Z, Ye Y, Cao H, et al. Clinical and dermoscopic manifestations of acute telogen effluvium. J Clin Dermatol. 2017;42(2):89-92.

2. Malkud S. Telogen effluvium: a review. J Clin DiagnRes. 2015;9 (9):1-3.
3. Veraldi S, Vaira F, Raia DD, et al. Telogen effluvium as first clinical presentation of dengue. G Ital Dermatol Venereol. 2017;152 (2):184-185. doi:10.23736/S0392-0488.16.05075-6

4. Rebora A, Ciccarese G, Drago F. Postfebrile telogen effluvium: hair fall after Rickettsia conorii infection. G Ital Dermatol Venereol. 2019;154(1):94-95. doi:10.23736/S0392-0488.17.05643-7

5. Xiong Q, Xu M, Li J, et al. Clinical sequelae of COVID-19 survivors in Wuhan, China: a single-centre longitudinal study. Clin Microbiol Infect. 2020. doi:10.1016/j.cmi.2020.09.023

6. Moreno-Arrones OM, Lobato-Berezo A, Gomez-Zubiaur A, et al. SARS-CoV-2-induced telogen effluvium: a multicentric study. $J$ Eur Acad Dermatol Venereol. 2020. doi:10.1111/jdv.17045

7. Hoffmann M, Kleine-Weber H, Schroeder S, et al. SARS-CoV-2 cell entry depends on ACE2 and TMPRSS2 and is blocked by a clinically proven protease inhibitor. Cell. 2020;181(2):271-280.e278. doi:10.1016/j.cell.2020.02.052

8. Liu J, Li Y, Liu L, et al. Infection of human sweat glands by SARS-CoV-2. Cell Discov. 2020;6(1):84. doi:10.1038/s41421-02000229-y

9. Turkmen D, Altunisik N, Sener S, et al. Evaluation of the effects of COVID-19 pandemic on hair diseases through a web-based questionnaire. Dermatol Ther. 2020;33:e13923. doi:10.1111/ dth. 13923

10. Kutlu Ö, Metin A. Relative changes in the pattern of diseases presenting in dermatology outpatient clinic in the era of the COVID -19 pandemic. Dermatol Ther. 2020:e14096. doi:10.1111/dth.14096

\section{Publish your work in this journal}

Clinical, Cosmetic and Investigational Dermatology is an international, peer-reviewed, open access, online journal that focuses on the latest clinical and experimental research in all aspects of skin disease and cosmetic interventions. This journal is indexed on CAS.
The manuscript management system is completely online and includes a very quick and fair peer-review system, which is all easy to use. Visit http://www.dovepress.com/testimonials.php to read real quotes from published authors. 